\title{
Towards Legged Amphibious Mobile Robotics
}

\author{
Chris Prahacs*, Aaron Saunders**, Matthew K. Smith*, Dave McMordie**, Martin Buehler** \\ * Mechanical Engineering \\ McGill University \\ Montreal, QC, Canada \\ \{chris.prahacs, matthew.smith\}@mail.mcgill.ca \\ **Boston Dynamics \\ Cambridge, MA, USA \\ \{aaron, mcmordie, buehler\} \\ @BostonDynamics.com
}

\begin{abstract}
New areas of research focus on bridging the gap between mobile robotics on land and at sea. This paper describes the evolution of RHex, a power-autonomous legged land-based robot, into one capable of both sea and land-based locomotion. In working towards an amphibious robot, three versions of RHex with increasing levels of aquatic capability were created. While all three platforms share the same underlying software, electronic and mechanical architectures, varying emphasis on aspects of similar design criteria resulted in the development of varied platforms with increasing ability of amphibious navigation.
\end{abstract}

\section{Introduction and Background}

Legged robots have long captured the imagination of researchers for the promise, motivated by observations of animal locomotion, for outstanding rough terrain mobility and versatility. Yet, artificial legged machines of the past were hard pressed to compete in terms of outdoor rough terrain mobility even with similarly sized tracked and wheeled vehicles. This is about to change, thanks to improvements in actuation, sensing, materials, computation, and most importantly, our understanding of locomotion. A product of these improvements and understanding has been RHex, a six-legged robot, [1, 2] which is currently the most agile and versatile untethered legged robot in existence (www.rhex.net). It was developed from 1999 - 2004 in an interuniversity collaboration between McGill and several US universities, with sponsorship from DARPA. RHex has continuously improved over the years, and we will refer to the many iterations that have not been waterproofed (but have been ruggedized considerably) as "Research-RHex". This paper takes Research-RHex as a point of departure and describes subsequently the RHex versions that were waterproofed and further ruggedized.

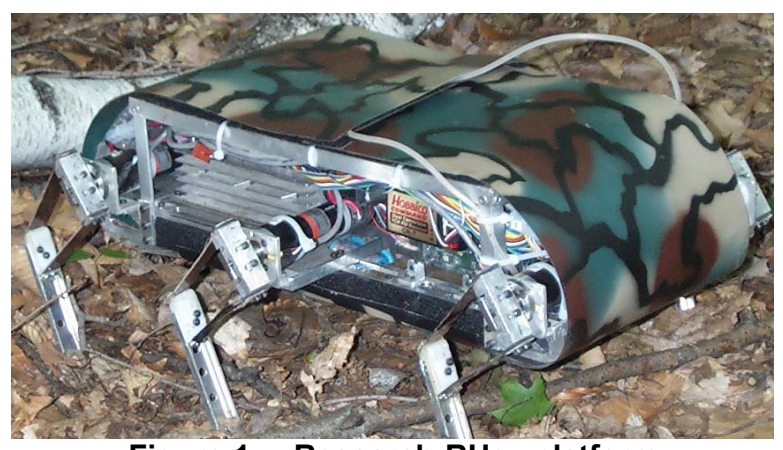

Figure 1. - Research-RHex platform

The current Research-RHex is a compact and agile hexapod robot able to walk and run up to $2.7 \mathrm{~m} / \mathrm{s}$ on flat terrain [3], traverse highly varied outdoor environments, climb slopes over 40 degrees, and traverse railroad tracks, brush and brambles. RHex's construction demonstrates that an exceptionally agile robot can result from the combination of simple compliant and recirculating legs, each with only one actuated degree of freedom, driven in a clocked, openloop manner [1].

The first RHex was constructed on an open aluminum frame with bent Delrin legs coupled directly to the motor/gear output shafts. Control was accomplished with an eight-card $\mathrm{PC} / 104$ computer stack (standard industrial computer format) running Proportional-Derivative servo and gait-generation software in a $1 \mathrm{kHz}$ loop. This simple embedded system controlled its actuators via a custom-designed motor control and breakout boards and a custom breakout board. Power was supplied from two $12 \mathrm{~V}$ sealed lead acid batteries. The first version of RHex weighed $7 \mathrm{~kg}$ [2] and was able to walk at about 0.5 $\mathrm{m} / \mathrm{s}$ for over fifteen minutes on one battery charge. It was able to demonstrate exceptional mobility over obstacles exceeding its body clearance. Yet failures were common, with fractures of the Delrin legs, motor shaft slippage and connector problems being the most frequent.

Design refinement reduced the mass of RHex to about $6 \mathrm{~kg}$, increased the run time to over 30 minutes 
and liberated much of the internal volume occupied by the $\mathrm{PC} / 104$ stack, motor drive and I/O card. Versions of this platform demonstrated stair climbing [4], pronking [5], bounding [6], flipping [7], pipe-traversal behaviours, and even a bipedal running mode [8]. A 3axis laser gyro and onboard vision system were later added to provide sufficient feedback for self-servoing abilities, including online autonomous gait parameter optimization [3].

As the mobility and performance of the research platform improved rapidly, it became apparent that the original open aluminum frame, even with the addition of a Lexan cover (see Figure 1), would not be sufficient for rigorous outdoor testing in uncontrolled environments. The platform now routinely encountered such hazards as rain, mud, sticks and sand, and often fell from heights greater than $30 \mathrm{~cm}$. Moreover, in an early display of legged robot versatility in 2001, RHex's body was sealed and demonstrated for the first time amphibious abilities. It walked from a sandy beach into a lake, swam using its unmodified legs on the surface, and walked back out on land over slippery rocks.

This experiment, combined with the RHex project's mandate to demonstrate increased ruggedness and reliability in all outdoor environments, initiated the development of three new successive robot designs: Shelley-RHex, Rugged-RHex, and AQUA. These three platforms, described in the sequel, each served to increase the platforms' waterproofing and/or ruggedness.

\section{Shelley-RHex}

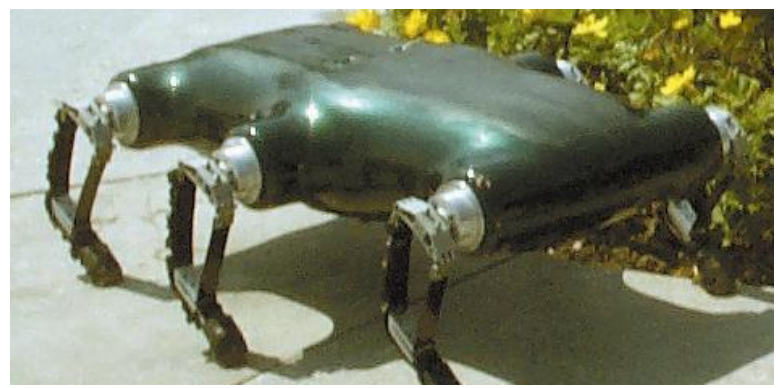

Figure 2. - Shelley-RHex platform

In order to protect the electronics from impact, dirt and moisture, a project was undertaken to replace Research-RHex's open frame with an enclosed shell. Loose specifications for the shell prescribed that it should withstand high impact loads while not significantly increasing the weight or size of the robot, and that it should be waterproof enough that the platform would be able to operate amphibiously.

\subsection{Shell Design}

Composites were chosen as the shell material because of their high strength-to-weight ratio as well as their amenability in conforming to complex curvatures. Specifically, carbon fiber was selected over Kevlar for its non-hydroscopic properties. Indeed, the final shape chosen for the shell was composed entirely of curved surfaces for several reasons:

1. They allow for the more even distribution of impact loads.

2. They reduce hydrodynamic drag while swimming.

3. They decrease the likelihood of the platform getting 'caught up' on obstacles during land-based operation.

4. They provide a pleasing aesthetic more reminiscent of biological organisms.

While the carbon fiber shell was being designed, hip sealing tests were performed using the research platform. Since the robot's 'hip' design at the time consisted simply of clamping a leg directly to the output shaft of the motor gearhead, small rubber orings had to be used to seal the rotating shafts, since standard rotary seals were not available in the necessary diameter.

However, while the shell design was underway, the hip was also redesigned to improve durability through the addition of a bearing supported shaft collar. The bearing increased the hips' robustness, eliminating shaft failures due to bending loads, while increasing the diameter of the hip's sealing surface enough to allow for the use of standard single-lip rotary seals.

Initially made from titanium to satisfy dynamic seal surface hardness specifications, later versions of the shaft collar were fabricated in stainless steel with no apparent reduction in performance.

In order to slide over the motor assembly shaft, an internal ' $\mathrm{D}$ ' shaped hole was cut using wire electrical discharge machining (EDM) through the full length of the shaft collar. The gap between the shaft collar and motor assembly shaft was designed at 0.005 " in order to allow for ease of assembly and because it was specified as the optimal gap (in terms of strength) for the removable 2-part acrylic glue selected to bond them. Threads were then added to the external end of the shaft collar in order to allow for disassembly. Removal of the shaft required heating the assembly above $250 \mathrm{~F}$ for several seconds and 'pushing' the motor assembly shaft out of the shaft collar using a modified bolt inserted into its threaded end.

The shell's 'one-piece' design necessitated the use of a removable body panel to replace batteries and provide access to the electronics. This panel was 
originally sealed using common weather stripping and screws to apply sufficient clamping force. As the shell was tested, however, this design was improved through the use of a hollow rubber extrusion for better sealing and quarter-turn fasteners to improve ease and speed of accessibility.

Other sealing issues arose through the need to use an external power switch, and from the antenna's interface with the shell. In the former case, an appropriate waterproof switch could not be found with sufficient amperage rating so a recessed standard automotive switch was used and sealed using a clamped elastomeric film. The antenna was sealed by simply smearing its mounting flange with silicone caulking during assembly.

While the shell provided the electronics with impact, debris and moisture protection from the environment, increasingly rigorous testing of the platform still subjected them to damaging vibrations. Neoprene dampers were used as an interface between the electronics and the shell in order to mitigate these effects.

While use of the shell increased platform length by 3 in. and width by 1 in., it actually became slightly thinner than the research version, thus increasing ground height and obstacle avoidance. This design tradeoff was achieved by splitting the PC/104 stack in two, and connecting the two halves using a ribbon cable. The shell's pleasing aesthetic combined with its impressive mobility impressed observers and potential investors.

\subsection{Performance}

Overall, the performance of the carbon-shell platform met expectations. It was able to operate in previously untenable environments (surface swimming) without sacrificing performance. However, for the developers, internal component access proved to be difficult and mold fabrication an expensive and lengthy process, limiting the ability of the developers to make design modifications and improvements while staying within budget limits and time constraints. The lack of flat surfaces inside the shell made the mounting of components very difficult, and the carbon fiber proved to be quite brittle. As such, repeated abuse during testing created cracks and holes that sabotaged its ability to operate in water. In addition, the shell proved to be too flexible in the region round the main seal, which limited its performance, as consistent clamping force and seal compression was not possible to achieve. Due to the tight packaging of the internal components, there was insufficient room for redesign through the use of local reinforcements.
These factors resulted in the under utilization of the developed platform. Due to the aforementioned costs of modifications to the shell design, the above issues were not addressed until the creation of the subsequent platform.

The broad design goals for the next platform were quite similar to those of Shelley-RHex, to develop a highly mobile platform that could walk or swim in most weather conditions. The material and sealing problems experienced with Shelley-RHex, the desire to improve ease of platform maintainability and design iteration, motivated differences in the emphasis and the details of the chosen development goals and constraints. What followed was a radically different platform, known as Rugged-RHex.

\section{Rugged-RHex}

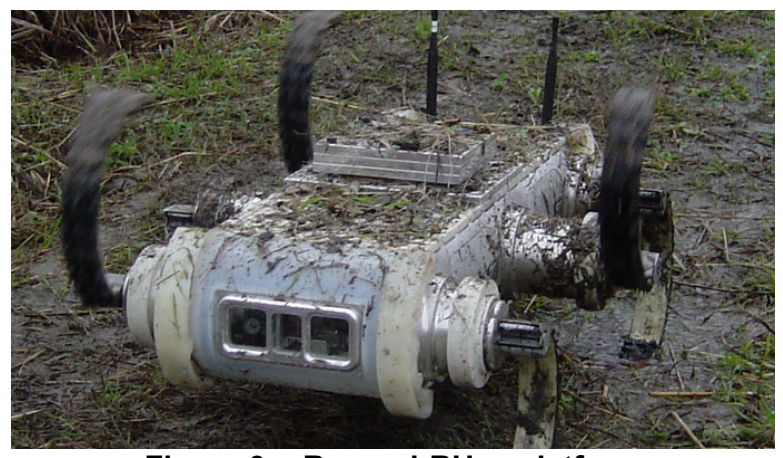

Figure 3. - Rugged-RHex platform

Rugged-RHex was designed in an effort to explore its application as a commercially viable, "backpackable" unmanned robotics product for the United States' military. This occurred in response to sponsor interest expressed after observations of the operational capabilities of previous versions of the RHex platform.

In an effort to produce a platform with the capability of participating in formal Operational Test Scenarios and other sponsored demonstrations and experiments, the following relevant specifications were provided to the designers:

- Platform of less than $10 \mathrm{~kg}$

- $2 \mathrm{~kg}$ payload capacity

- $2 \mathrm{~km}$ travel distance

- 6 hour mission duration

- Tele-operative communication via IEEE 802.11b (commercial wireless Ethernet)

- $\quad$ Switched video sources for two internal (day and night) cameras

- Mechanical mounting rail for payloads

- Simple battery exchange mode

- Water resistant / operation in rain 
While the specifications did not specifically require submersible operation, a decision was made by the design team to combine the new platform's development with that of one capable of such underwater mobility, thus further increasing its mobility spectrum. The target set for waterproof depth was $10 \mathrm{~m}$.

Other additions were made to the platform's specifications in which emphasis was placed on 'enduser' requirements. The decision was made to build a platform that made use of standard military issue batteries (specifically SINCGAR radio batteries), and to focus on field maintainability through the use of field replaceable units for high risk and maintenance items. In this regard, a tool-less leg exchange mechanism and battery carriers were developed, and external access to individual motor assemblies was provided for ease of replacement. Improved impact resistance, and an improved user interface (rugged, portable, reliable and user friendly) were also targeted.

\subsection{Shell and Frame}

The general layout and overall platform dimensions were primarily driven by both the size of the selected motor/gearhead/encoder combination and the batteries. The decision to conform to a battery using a standard military footprint had a particularly significant impact upon the design, resulting in a larger (and resultantly heavier) platform than originally anticipated. In fact, the legs had to be lengthened in order to overcome the decrease in ground clearance that resulted from the thicker body.

Using lessons learned from the Shelley-RHex platform, the design evolved from the inside out. In this way the expected increase in platform size was minimized without requiring an effort to develop additional custom electronics. To improve the ease of platform maintenance and upgradeability, a compromise was made between exoskeleton and endoskeleton design paradigms. The resulting platform's structural integrity came primarily from the external shell. However, internal ribs were used to increase platform stiffness and to provide mounting points for the batteries and other electronic components, keeping them away from the shell's impact surfaces, while allowing easier revisions to component mounting. See Figure 4. A more modular shell design allowed access to the electronics from any side of the robot without having to disassemble the entire system, and enabled materials for each region of the shell to be chosen according to that component's specific requirements.

A standard military interface was provided for payload attachment. A USSOCOM aluminum rail was copied in Nylon (to reduce weight) and fastened to the top of the platform.

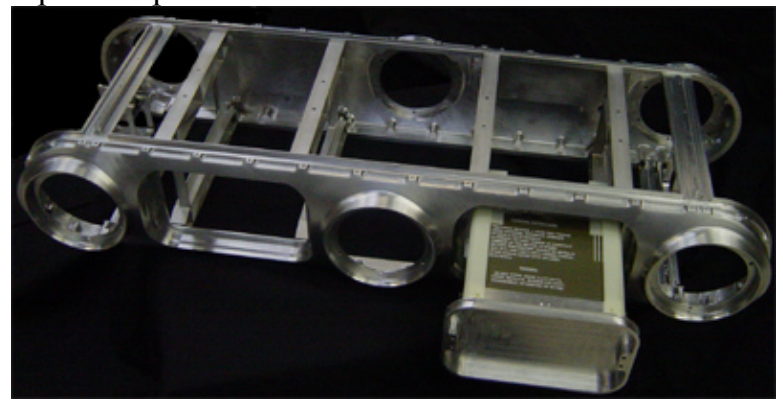

Figure 4. Rugged-RHex frame - shown with battery in carrier

The desire to reduce complexity and weight resulted in the choice of a single-cavity sealing concept versus one incorporating multiple individually sealed modules. In addition, an effort was made to create multifunctional components wherever possible. One example of this was the main side frame, which served not only as the primary source of structural rigidity for the platform but also as a heat sink for heat producing electronic components. See Figure 4. In this way the component helped move heat generated inside the sealed body to the external environment, both extending the platform's operational capabilities and reducing the danger to the internal components. The choice to use aluminum in this context was driven by its high strength-to-weight ratio as well as its high thermal conductivity. The material specifically chosen was AL7475. Though not quite as strong as AL7075, its thermal conductivity is slightly higher, and it is has a higher fatigue stress limit. In order to better distribute the impact loads transferred by the hips, the side frame was designed with a face curved laterally outwards at mid-height. (See Figure 7 for a detailed view)

Experience with the brittle carbon fiber shell forced a reevaluation of material choice for the impact-bearing shell components. Composites were rejected as it was learned that even with non-brittle materials like Kevlar, impact absorption comes at the price of delamination, which would have compromised the watertight cavity. Metals were also rejected due to their inability to absorb impact energy. Polymers in general seemed the best choice due to their energy dissipation properties as well as their tendency to deform plastically rather than catastrophically, thus ensuring the maintenance of a watertight seal even when exposed to very concentrated loads. Dupont's Zytel Super Tough Nylon ST801 was chosen for its extremely good notched-impact resistance, as well as for its low friction coefficient (easing the traversal of obstacles). 


\subsection{Actuators}

Based on the initial estimate of the overall platform weight breakdown, a comprehensive review of suitable DC motors and gear systems was made. A list of possible candidates was generated, and it was noted that it would be preferable to maintain the same Maxon RE20/GP32 motor/gearhead/encoder combination as used in previous iterations of RHex, with the only exception being the move from an $18 \mathrm{~V}$ motor to a $42 \mathrm{~V}$ version. This change allowed the use of a higher specific energy Li-Ion battery chemistry (vs. a NiMH chemistry) by decreasing motor current requirements while maintaining the same power output. (Today's Li-Ion batteries cannot safely output currents at levels comparable to NiMH batteries.)

However, an increase in platform mass over initial estimates $(\sim 30 \%$ greater $)$, resulting from machining thickness requirements and additional sealing components and features, along with an increase in leg length and the need to be able to carry a $2 \mathrm{~kg}$ payload, resulted in the replacement of these motors with larger Maxon RE36 models. The originally selected RE20/GP32 actuators demonstrated insufficient torque to achieve desired performance. As well, due to the fact that these motors were being driven constantly at or close to maximum torque, failures due to overheating (rotor deformation) were common. Although the larger RE36 actuators corrected these problems they also contributed to a further increase in platform mass.

To further reduce the chances of motor failure, heat sinks were developed that enveloped the motor housing and were thermally coupled to the hip assemblies (using thermally conductive paste), where heat could be dissipated externally. In addition, a motor dynamometer was built to measure the relationship between internal core temperature, motor current, and motor case temperature. A control model was developed to limit motor current when necessary to protect it from overheating [9]. Temperature sensors bonded to the motor cases provided the case temperature data required by the core temperature model.

Still, there are circumstances where the operator may need to push the motors above the safe limits to complete a specific task. If this occurs, the focus then turns to maintainability. To this end, the robots' hip assemblies could be exchanged individually and detached externally by removing the seal carrier and disconnecting the motor harness. This procedure required one person approximately ten minutes and only one standard tool. Since all hips were of identical design even on opposite sides of the platform, components in the maintenance kit were reduced and the replacement process was further simplified.

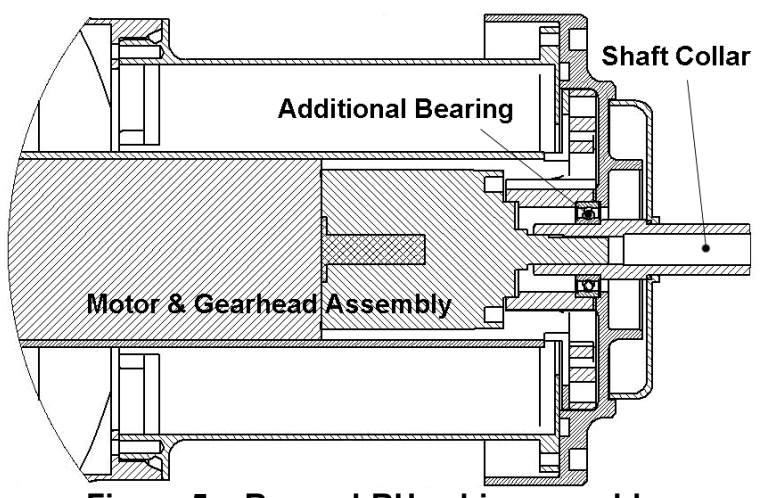

Figure 5. - Rugged-RHex hip assembly

The robotic hip shown in Figure 5 is composed of five major components: motor assembly, bearing, hip, output shaft collar, and heat sink.

\subsection{Sealing}

The most significant challenge faced was sealing the individual body and frame components to protect the internal electronics. This was achieved using a mix of available consumer products and several custom designed seals. The complicated geometry on the platform required ten unique seals, a total of thirty-six individual seals. Each hip assembly contains one dynamic rotary seal on the main hip shaft and three static o-ring face seals. The main body seal is a custom designed compression face seal. The seals on the removable battery carriers are custom static radial compression seals.

Standard consumer seals were used wherever possible. The hip extension was sealed to the main side frame using a crush style standard size o-ring. A similar seal was used on the removable shaft extension plugs. Simple static gland o-ring face seals were used on the antenna mounts and hip extension caps. Gland size was determined based on manufacturer specifications [10]. Nitrile rubber was the selected oring material, due to its compatibility with salt and fresh water.

In static seal applications the recommended squeeze for most elastomers is $33 \%$ [10]. This amount of squeeze provides sufficient compression without permanent seal deterioration. Additionally, the minimum seal squeeze, regardless of cross section, should be $0.2 \mathrm{~mm}$ as with a very light squeeze most elastomers will take on $100 \%$ compression set (percent of original deflection). A compression value of $23 \%$ or $0.9 \mathrm{~mm}$ was selected for the main body seal. Another variable to be considered is gland fill, the percentage of the gland area occupied by the seal cross section. Recommended values are between 60 and $85 \%$. A gland fill volume above this would not leave enough room for compression or thermal expansion effects. The final body seal cross section was designed to fill 
$75 \%$ of the seal boss, thin extrusion features were added to ensure side-to-side alignment within the boss. As seal elongation reduces the cross section, to maintain proper gland fill it was important to ensure the 3D body seal was not deformed upon final installation. This was accomplished by attention to sizing of the length of the seal.

A Shore A $40 \pm 5$ durometer silicone compound was chosen for the body seal because of its broad temperature range and common availability in short run fabrication facilities. This is much softer than standard o-ring hardness, which is often in the Shore A 70 to 80 range. Softer sealing materials flow more easily into any microfine grooves and imperfections of machined parts. This was of particular interest when working with machined plastic body components, as the surface finish of nylon is often not ideal. Additionally, softer seals require far less pre-load to ensure good seal contact for low-pressure applications. This was an important consideration for the Rugged RHex platform, as it was designed for land and shallow water applications, where the compression seal force due to external pressure will be low. The final seal cross section for the main body seal is shown in Figure 6.

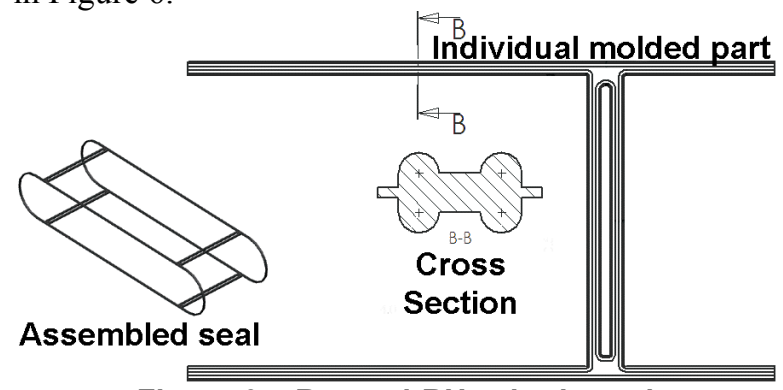

Figure 6. - Rugged-RHex body seal

The external surfaces of the platform are comprised of six major sections, with two each of side frames, top panels, and end panels. The side frame geometry was designed to provide a continuous seal path around its perimeter surface. The most complicated part of the seal is the " $T$ " joint between the side frame and the mate line between any two shell pieces. The seal design is an assembly of four " $\mathrm{H}$ " shapes into simple rectangles (picture the curved end shells flatted out). Figure 6 shows the planar design of the seal assembly. The ribs shown in the section view create the single bead face seals around each of the rectangular seal sections.

Molds required to fabricate 3D seals are complex and expensive compared to planar seals. To reduce mold costs, the body seal was fabricated using a simple planar " $H$ " shape, where four of these shapes are spliced together to form the final 3D seal, shown in Figure 6. The splicing is accomplished using specialized adhesives that maintain similar properties to the seal material after curing. Using a multi-piece assembled seal resulted in a $90 \%$ reduction in mold cost.

The two battery compartments are sealed using simple radial compression seals, shown in Figure 7. The battery compartments pose one of the highest seal liabilities as they are frequently removed and replaced from the single cavity body. A radial compression seal was chosen over a standard o-ring style face seal for higher reliability in dirty environments. The seal cross-section, as shown in Figure 7, was extruded in a continuous fashion and spliced into loops to fit the battery door seal channel. The three-lip seal design wipes the surface as it is removed and replaced from the boss, increasing the chance of a good seal. This self cleaning feature was important as in the operational environment there is no way to ensure that both the seal and its mating surface remain free of dirt. The same material and design principles described above in the body seal section were used for the battery door seal. There were some challenges in finetuning the amount of seal compression to ensure a good seal without making the sliding fit too tight.

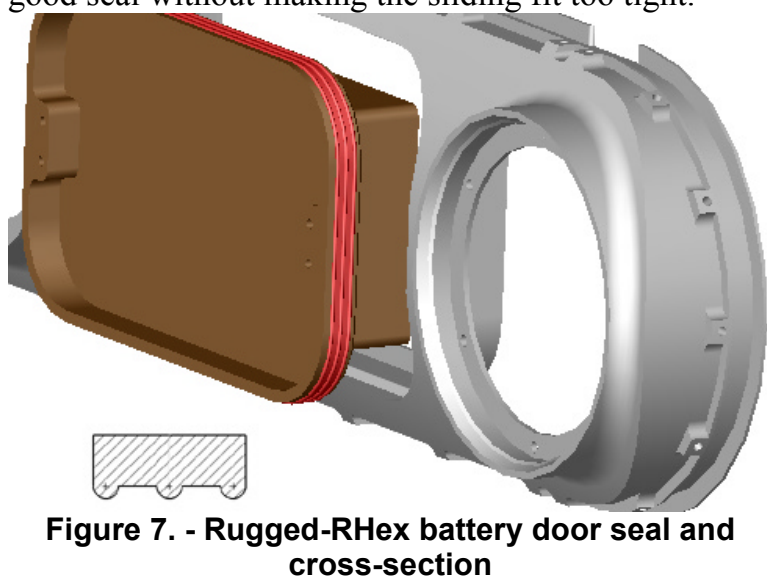

The platform has six dynamic shaft seals, one on each drive shaft. The seals are spring-energized, graphite filled, Turcon ${ }^{\circledR}$ PTFE from American Variseal. The graphite filled PTFE material is well suited for low friction, dry running, applications where little or no lubrication is available. The graphite fiber greatly improves wear resistance, at the expense of flexibility.

Because the dynamic seal boss was machined Nylon an external o-ring was selected to prevent seal rotation relative to the boss, and to reduce any chance of fluid bypassing the seal altogether.

Several problems were encountered with these hard graphite filled seals. Although the material properties of the graphite filled PTFE were ideal for low friction the hard material was susceptible to 
surface damage during frequent assembly and disassembly of the prototype system. This seal type was particularly sensitive to seal surface diametric tolerance and finish. Additionally, the seals did not hold up well against fine grit, such as sand, commonly encountered in outdoor environments.

For underwater applications a commonly available, spring-energized, Nitrile oil seal was evaluated. Because of the durable properties of Nitrile, the seals were able to withstand more frequent maintenance. The downside was their poor wear properties in dry running terrestrial environments. Future platform development will require further testing of seal materials in order to find a seal adequate for both dry running terrestrial applications and higher pressure aquatic environments.

\subsection{Communications}

Substantial effort was invested in developing an integrated IEEE $802.11 \mathrm{~b}$ communications link for the platform, providing both video and control links through a single channel. However, initial testing showed abysmal performance in brush, and at best only short-range performance in open-environments. As well, video compression and decompression resulted in unacceptable video latency (due in part to the fact that the embedded processor on the robot was not appropriate for this application). For these reasons, the IEEE $802.11 \mathrm{~b}$ system was replaced with a $1 \mathrm{~W}, 900 \mathrm{MHz}$ bi-directional radio modem link combined with a $2 \mathrm{~W}, 1.7 \mathrm{GHz}$ analogue video transmitter. Though less integrated, this system provided increased range and improved penetration in brush environments. As well as providing improved range and reliability, the new system provided more graceful degradation of both video quality and control bandwidth when reaching the limit of transmission range.

\subsection{Batteries}

One major challenge when selecting battery technology for military applications is their availability in the standard supply chain. The batteries selected for the Rugged-RHex platform were standard SINCGAR radio batteries, available as either NiMH (BB390) or LiIon (BB 2590) units. By using batteries commonly available in the field, charging and replacement was simplified. Two identical battery compartments accept tapered carriers that automatically align the batteries with the internal mating connectors. The battery carriers slide directly into mating features in the main frame, providing a radial compression seal when fully inserted. Radial seal pressure was maintained using a tool-less quick release door latch.

\subsection{Legs}

The shape of the legs mirrored those used on the research platform, as the half-circle shape had demonstrably aided platform mobility over obstacles and stairs. One difference, however, was their width, which was increased by $35 \%$ in order to increase their lateral stiffness and to decrease their thickness. Increased lateral stiffness was desired in order to more successfully climb inclines at an angle. It was hoped that a reduction in leg thickness would result in decreased interlaminar shear stresses, a common source of failure in previous versions.

To increase the mean-time-between-failures (MTBF) of the legs the material was changed from Eglass to S-glass, which can store more strain energy. The glass/resin system was specifically selected for maximum toughness, under the constraints of the production facilities available to the design team. The new legs were a complete success - no leg material failures were observed during hundred of operational hours. Leg stiffness, critical to the performance of the more dynamic robot behaviors, had to be retuned (by adjusting the composite lay up and resulting stiffness) to accommodate the final mass of the robot.

A tool-less leg exchange mechanism was designed to allow rapid leg detachment for in field maintenance and transportation By using a simple thumb activated over center cam mechanism, leg replacement can be done in a very short period of time without tools. To enable tool-less actuation the activation force and lever area was based on realistic adult thumb-finger forces [11]. In hindsight, due to the exceptional durability of the legs, this feature turned out to be less important than originally thought.

The 2-part acrylic adhesive originally chosen for the Research-RHex and Shelley-RHex platforms proved to lack the strength required to endure the higher torques output by the new, larger motors. As a result, much time was spent repairing the shaft collar assemblies. In this context, there was a distinct tradeoff between using a stronger adhesive that would require less maintenance vs. using a weaker, 'removable' adhesive. We chose to use the weaker, 'removable' adhesive for the simple fact that the 6week lead time on motor assembly components necessitated their recycling whenever possible in the event of motor failure.

\subsection{Performance}

The increase of the platform mass that goes along with any hardening effort had several repercussions in terms of performance. As the platform became heavier, impact forces resulting from falls from any height became larger, thus increasing the wear on components. The increase in size and 
mass also made the platform less portable and decreased the product's appeal in the context in which it was designed. While the decision to conform to standard military batteries proved to be a selling point with evaluators, the penalty was paid in terms of size and energy density compared with more advanced alternatives.

In spite of these challenges the platform proved to be a general success overall. It was tested and proven waterproof to $5 \mathrm{~m}$, though observed deflections in the Zytel top and bottom shell components prompted the team to limit testing to that depth. The platform proved itself more durable and reliable in operation than previous iterations on land, and for surface swimming, and extended operational regimes (when ballasted) into underwater operation.

\section{AQUA}

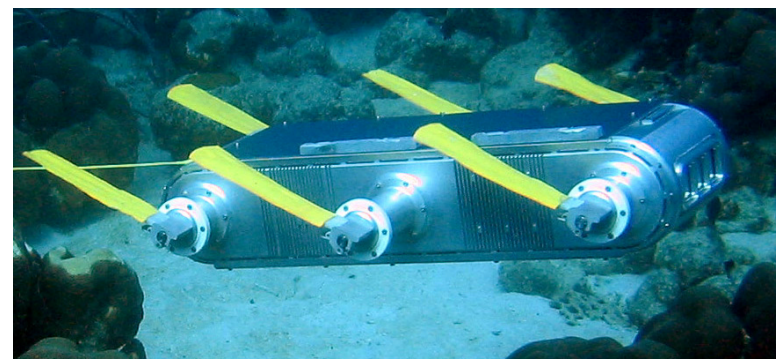

Figure 8. - AQUA platform

Based upon the Rugged-RHex platform, the goal of the AQUA project was to develop a platform and computer-vision system capable of generating three-dimensional maps of coral reefs (www.aquarobot.net). The platform was required to be capable of walking on both land and in water, and of swimming to a depth of $10 \mathrm{~m}$. Development of the AQUA platform was made possible by the generous support of the Canadian Inst. for Robotics and Intelligent Systems (IRIS).

While the general mobility requirements remained similar to Rugged-RHex, the primary goals in developing the platform were to minimize costs, and increase the operating depth. Additionally, it was important to optimize the platform for operation primarily in the underwater regime without sacrificing mobility on land. Cost and time constraints necessitated the reuse of the body seals and batteries. However, all other custom components were revised or redesigned to achieve these goals. This allowed for lessons learned from the construction and operation of Rugged-RHex to be incorporated into the new design.

\subsection{Shell}

Two main factors drove the redesign of AQUA's shell components, reduced cost, and enclosed volume. Initial underwater testing with the previous Rugged-RHex platform required a significant amount of ballast to be added in order to achieve neutral buoyancy. To reduce the amount of required external ballast, the platform's enclosed volume was reduced, and machining operations, previously used to reduce weight, were eliminated. With the reduced body volume and simpler parts, the mass of external ballast required was decreased by $40 \%$. Overall, the weight of the robot when ballasted for neutral buoyancy was reduced by $10 \%$, allowing improved on-land performance, and increased portability.

To reduce manufacturing costs both the number of machining operations, and the use of expensive Zytel polymers was addressed. To reduce machining costs, the load-bearing compound curves from the side panels of the robot (see Figure 7) were replaced with a flat surface reinforced by internal ribs. In addition, the previous platform used a number of components that were designed with very thin sections $(\sim 1 \mathrm{~mm})$ to save weight. Thin cross-sections, leading to vibration during manufacture, resulted in high part costs as suppliers adjusted for increased machining time and test parts. To eliminate vibrations during machining, the cross sectional thickness was increased by $\sim 25 \%$, wherever possible. Also, the internal pockets and ribbing of the frame panels were designed to be machined with only two tools, reducing the setup time compared to the previous design. Overall, the cost of manufacturing the components with similar features was reduced by over $25 \%$.

Finite-element-analysis was used as an aid in two particular areas of the design, firstly, to optimize the strength of the top and bottom shell components, and secondly to improve the robustness of the hip assembly. FEA enabled the selective incorporation of reinforcing ribs of varying widths where necessary, optimizing strength in critical areas. While these features did somewhat increase the costs of manufacturing, the need to maintain internal clearance for electronics was deemed to be more important than the added expense. To withstand the pressure when operating at greater depths, the Zytel shell components were replaced with 7475 aluminum. 

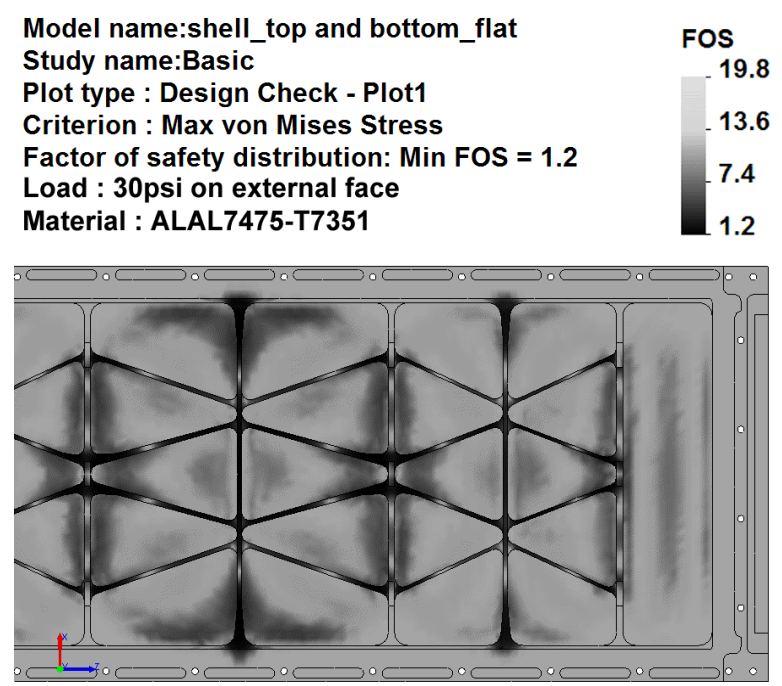

Figure 9. - Illustration of FEA analysis of top shell

Secondly, FEA was used in the redesign of the hip and motor assembly. Analysis enabled changes to significantly increase the ability of the hip to withstand impact loads, while reducing the number of components through integration. Operation of the Rugged-RHex platform had shown that the interface between the shaft collar and gearhead output shaft was unreliable. Analysis of the assembly indicated that shear and bending stresses were too high for the bonding agent selected. The robustness of the bond was increased with the selection of an optional larger output shaft ( $8 \mathrm{~mm}$ vs. $6 \mathrm{~mm}$ ), and a different bonding agent suited for a tighter fit between the components.

\subsection{Actuators}

In order to maximize the power autonomy and mobility of the platform, careful consideration was given to motor selection. The previous generations of platforms were limited by the amount of available torque, and by the overheating of motors. To optimize the output torque and speed for this application, a battery-amplifier-motor-gear model was developed to evaluate the available alternatives.

Using the model, it became evident that the internal resistance of the motors would limit torque production when battery currents were above $50 \%$ of their peak. Either increased battery voltage or decreased motor resistance was required to allow maximum torque production. By modeling numerous combinations of motors and gear ratios, a short list of potential candidates was generated. Long supplier lead-times meant that only in-stock models could be used for initial tests. The first motors obtained came with a gearhead ratio $1 / 2$ of the desired speed. Performance in the water was adequate, but operation on land was compromised by the lack of leg speed, pending the arrival of motors with the preferred gears.

\subsection{Control and communications}

Remote operation in an aquatic environment provided a number of additional challenges. Underwater, the robot has six navigational degrees of freedom, vs. only two on the ground. Currently, directional control of the robot can only be accomplished by low-level, direct input of pitch, roll, yaw, and thrust. The operator uses images for control decisions, and hence a high-bandwidth link is required. As with the Rugged-RHex platform, the AQUA operator interface also provides information about robot health, and operational status. The future addition of an IMU would allow for software-based directional correction for perturbances, greatly simplifying the job of the operator.

Since existing underwater wireless communications tend to be limited to low bandwidths (e.g. 19200 baud for ultrasonic modems) a tether was required to relay visual data. However, even when slack at the operator's end, tethers can greatly affect the operation of the robot due to their inertia, buoyancy, and drag [12]. To reduce these effects, the tether selection needed to consider diameter, and tensile strength. To eliminate the need for power wires in the tether, the AQUA platform also uses onboard batteries, and is capable of operating for more than two hours at a time. To further reduce the number of communication channels, a multiplexer was selected that combined several channels of simultaneous twoway data, and three video streams onto a single optical fiber. With this system, a single-fiber tether of only $3 \mathrm{~mm}$ in diameter was used to control the platform from over $200 \mathrm{~m}$ away.

\subsection{Materials}

Operation in a salt water environment required careful selection of materials and surface treatments to reduce or eliminate galvanic corrosion caused by dissimilar metals. This is an especially important consideration for fasteners given that their shape and interface with components harbor many crevices. Since it was not cost effective to use aluminum fasteners, stainless steel ones were used. However, stainless steel is subject to crevice corrosion where two components meet. To mitigate this effect, silicone grease was used to fill the threaded holes, displacing any air that could become trapped during assembly and allow water to enter the threads when at depth.

Other corrosion fighting measures included the anodizing of all aluminum parts, powder coating of surfaces potentially at risk for scratches, the use of sacrificial zinc anodes, as well as thorough and immediate rinsing of the platform in fresh water to remove electrolytes after each use. 


\section{Conclusions:}

We described a succession of RHex robot designs that increasingly span the transition from land to water, and improve the platforms ruggedness. Lessons learned in terms of material selection, battery technology, actuation, cost, and shape selection have been carried over into subsequent designs, from Research-RHex to Shelley-RHex to Rugged-RHex to AQUA. However, due to different sets of objectives, the different platforms still occupy local peaks of performance. Research-RHex is the lightest and fastest platform, with a vision system and the most sensors for advanced controllers and some autonomy. RuggedRHex is still the only truly amphibious platform of the RHexes, with its ability to operate on land and on the water surface without modifications. AQUA is the most waterproofed platform, being designed for neutral buoyancy and maximized with its flippers and its body for diving underwater. Future versions of RHex could further expand the platform's versatility. For example, legs could be developed that are suitable for land mobility and at the same time provide thrust for underwater and surface swimming. Buoyancy control could enable the platform to linger or walk on the bottom of the sea, then dive underwater, and float on the surface, while maintaining a low mass suitable for high performance terrestrial locomotion.

\section{Authorship:}

The authors wish to note that the order of authorship is not indicative of the importance of their contributions to the project.

\section{Acknowledgements:}

The authors of this paper would like to thank the entire Research RHex team, under leadership of Professor Daniel Koditschek at University of Michigan for the development of the Research RHex platform and the RHexLib software, which runs all versions of RHex. We also acknowledge DARPA for all RHex funding (with the exception of AQUA), and for generously permitting us to use Rugged RHex as the first platform to successfully demonstrate underwater mobility in the AQUA project.

The authors of this paper would also like to thank the following people for their invaluable contributions to these projects. Without their enthusiastic efforts, development of these platforms would not have been possible:

Christine Georgiades and Shane Saunderson for the development and testing of the AQUA flippers;
Evgeni Kiriy for the design of the custom power supply for Rugged/AQUA; Jean Collin, Grant Smedley, Mathieu Bernier, and Thomas Lavertu for the development of the shell for Shelley-RHex; Edward Moore for his supervision of the development of Shelley-RHex; Al Rizzi for help with the Rugged RHex radio modem communication software; Joel Weingarten for assistance with tuning Rugged-RHex gaits; Nadim El-Fata and Don Campbell for the design of custom electronic hardware; Demetri Giannitsios for the design of the tool-less leg attachment mechanism; John Sheldon for assistance with manufacturing and assembly; James Andrew Smith for his assistance with various electrical aspects of the projects.

[1] M. Buehler, U. Saranli, and D.E. Koditschek, "Single actuator per leg robotic hexapod" McGill University, The Regents of the University of Michigan: USA, 2002, US Patent 6,481,513.

[2] U. Saranli, M. Buehler, D.E. Koditschek. "RHex: A Simple and Highly Mobile Hexapod Robot", The International Journal of Robotics Research 20, July 2001, pp. 616-631.

[3] J. D. Weingarten, G. A. D. Lopes, M. Buehler, R. E. Groff, D. E. Koditschek, "Automated Gait Adaptation for Legged Robots.” IEEE Int. Conf. On Robotics and Automation (ICRA) Vol. 3, New Orleans, LA, April 2004, pp.2153-2158

[4] E. Z. Moore, D. Campbell, F. Grimminger, and M. Buehler. "Reliable Stair Climbing in the Simple Hexapod 'RHex'." IEEE Int. Conf. on Robotics and Automation (ICRA) Vol. 3. Washington, DC, May 2002, pp. 2222-2227. [5] D. McMordie and M. Buehler. "Towards Pronking with a Hexapod Robot." 4th Int. Conf. on Climbing and Walking Robots, Karlsruhe, Germany, September 2001.

[6] D. Campbell and M. Buehler. "Preliminary Bounding Experiments in a Dynamic Hexapod." Experimental Robotics VIII, 2003, pp. 612-621.

[7] U. Saranli, D.E. Koditschek. "Back Flips with a Hexapedal Robot." IEEE Int. Conf. On Robotics and Automation (ICRA) Vol. 3, Washington, DC, May 2002, pp. 128-134.

[8] N. Neville, M. Buehler, "Towards Bipedal Running of a Six Legged Robot." 12th Yale Workshop on Adaptive and Learning Systems, May 2003.

[9] D. McMordie, C. Prahacs and M. Buehler, "Towards a dynamic actuator model for a hexapod robot." IEEE Int. Conf. on Robotics and Automation, Taipei, Taiwan, 2003. [10] Parker O-Ring Handbook, 2001 Edition, Catalogue ORD 5700A/US

[11] K. H. E. Kroemer, Engineering Physiology: Bases of Human Factors/Ergonomics, John Wiley \& Sons; $3^{\text {rd }}$ edition (July 25, 1997), ISBN 0471287989, p. 118

[12] T. W. McLain and S. M. Rock, "Experimental Measurement of ROV Tether Tension." Proceedings of IEEE Intervention/ROV '92, San Diego CA, June 1992, pp. 291-296 